\title{
Relevance, Influence and Threat of Wireless Ad-Hoc Network
}

\author{
Ahlal H Montaser Mohamed, Amamer Khalil Masoud Ahmidat, \\ Alhamali Masoud Alfrgani \\ Department of Computer Sciences \& Information Technology, 'Sam Higginbotom university of Agriculture, \\ Technology \& Sciences Allahabad India'
}

\begin{abstract}
In these days, we have seen a rapid expansion in the field of mobile computing due to widely available wireless devices. However, current devices, applications and protocols are completely focused on cellular or wireless local area networks A mobile ad hoc network is an autonomous collection of mobile devices (laptops, smart phones, sensors, etc.) that communicate with each other over wireless links and co-operate in a distributed manner in order to provide the necessary network functionality in the absence of a fixed framework. Ad hoc structure does not require an access point, it is easy to setup, especially in a small or temporary network. Each node in the network forwards the packet without the need of central administration. In ad hoc network, node acts as a router to send and receive the data. An advantage of the system is robustness, flexibility and mobility. This paper is focused on the applications, advantages and challenges of ad hoc networks.
\end{abstract}

\section{Introduction}

Mobile Ad-hoc NETWORK (MANET) is a collection of mobile devices communicating with each other without help of any centralized administration. The devices in MANET can move freely with seamless connectivity and form a self-organized network. MANET doesn't need any existing communication infrastructure. MANET is useful in military communication and other specialized fields such as disaster management and recovery, emergency services, and environment monitoring, etc. Military applications cannot rely on fixed infrastructure based communication services in battlefield but MANET is used to quickly selfconfigure the network and communicate with each other. In emergency services, MANET is used for search and rescue operations and replacement of fixed infrastructure in case of earthquakes, fire etc. There are various factors like scarcity in network resource, dynamic nature of applications, unstable links and topology, infrastructure less architecture, mobility of nodes etc., affect the performance of MANET. The communication over a dynamic environment like a mobile wireless network is difficult than communicating with a static channel because the link reliability, delay and packet loss are not determined. The rapid progress of mobile computing becomes a powerful trend in the development of Information Technology (IT) as well as commerce and industry fields. The next generation computing is moving towards the era of mobile cloud computing that integrates the cloud computing into the mobile environment and overcomes obstacles related to the performance. The nature of mobile cloud computing makes usage of mobile ad-hoc networks the easiest solution for the interconnection of devices. Mobile ad-hoc network consists of mobile nodes connected via wireless link without any centralized network infrastructure. Furthermore, nodes can move freely in the area which results in the rapid change in wireless network's topology. Hence, the dynamic change in topology causes frequent disconnections which result in large packet loss. When a route breaks, the discovery of a new route will most likely introduce delays, which will affect the performance of the network. One of the major challenges in ad-hoc networks is the security of connections between hosts in the network. With free-space radio transmission in the wireless environment it is fairly easy for a malicious host to eavesdrop on a communication session. This could lead to unauthorized access, information theft, interference, jamming and service degradation. Due to the multi-hop nature of ad-hoc transmissions, it is a very difficult to even detect such intrusion. The field of security for ad-hoc networks is at a very premature stage and this issue has to be thoroughly studied before ad-hoc network systems can be practically deployed in real world applications.

\section{Manet Concept}

A mobile ad hoc network is a collection of wireless nodes that can dynamically be set up anywhere and anytime without using any pre-existing network infrastructure. It is an autonomous system in which mobile hosts connected by wireless links are free to move randomly and often act as routers at the same time. The traffic types in ad hoc networks are quite different from those in an infrastructure wireless network, including:

\subsection{Peer-to-Peer}

Communication between two nodes which are within one hop. 


\subsection{Remote-to-Remote}

Communication between two nodes beyond a single hop but which maintain a stable route between them. This may be the result of several nodes staying within communication range of each other in a single area or possibly moving as a group. The traffic is similar to standard network traffic.

\subsection{Dynamic Traffic}

This occurs when nodes are dynamic and moving around. Routes must be reconstructed. This results in a poor connectivity and network activity in short bursts.

\section{Applications Of Ad Hoc Networks}

With the increased number of lightweight devices as well as evolution in wireless communication, the ad hoc networking technology is gaining effort with the increasing number of widespread applications. Ad hoc networking can be used anytime, anywhere with limited or no communication infrastructure. The preceding infrastructure is fancy or annoying to use. The ad hoc network architecture can be used in real time business applications, corporate companies to increase the productivity and profit. The ad hoc networks can be classified according to their application as Mobile Ad hoc NETWORK (MANET) which is a self-arranging infrastructure less network of mobile devices communicated through wireless link. Wireless Sensor Network (WSN) consists of autonomous sensors to control the environmental actions. The importance of ad hoc network has been highlighted in many fields which are described below:

\subsection{Military area}

An ad hoc networking will allow the military battleground to maintain an information network among the soldiers, vehicles and headquarters.

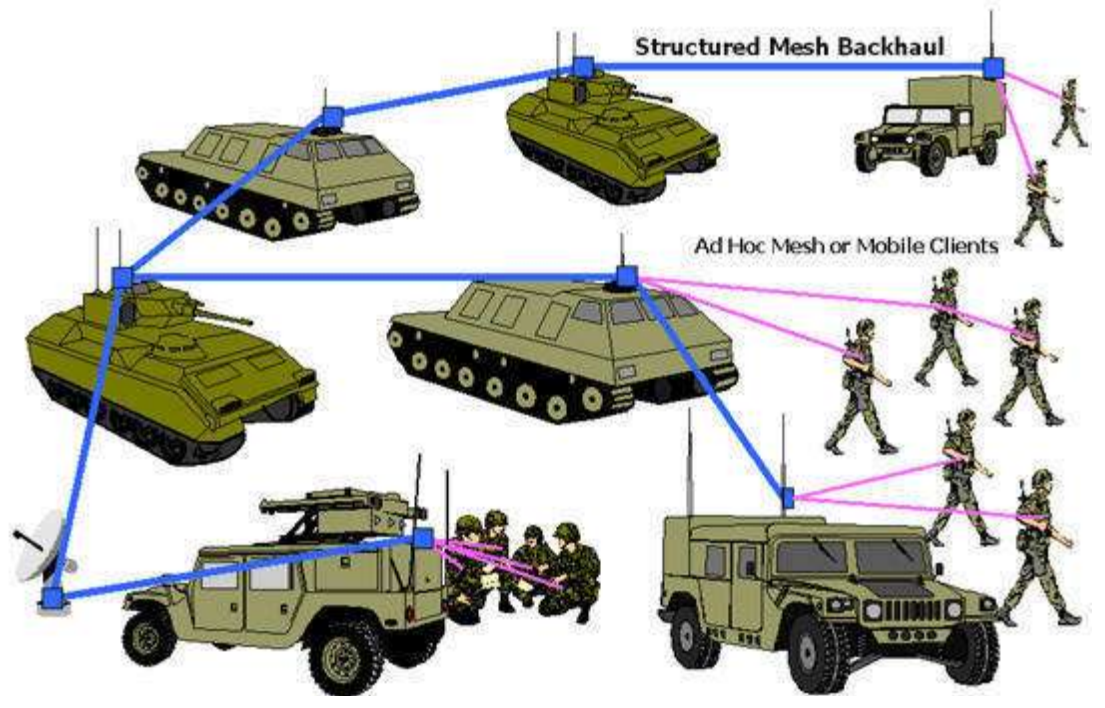

\subsection{Personal area network}

A personal area network is a short range, localized network where nodes are usually associated with a given range.

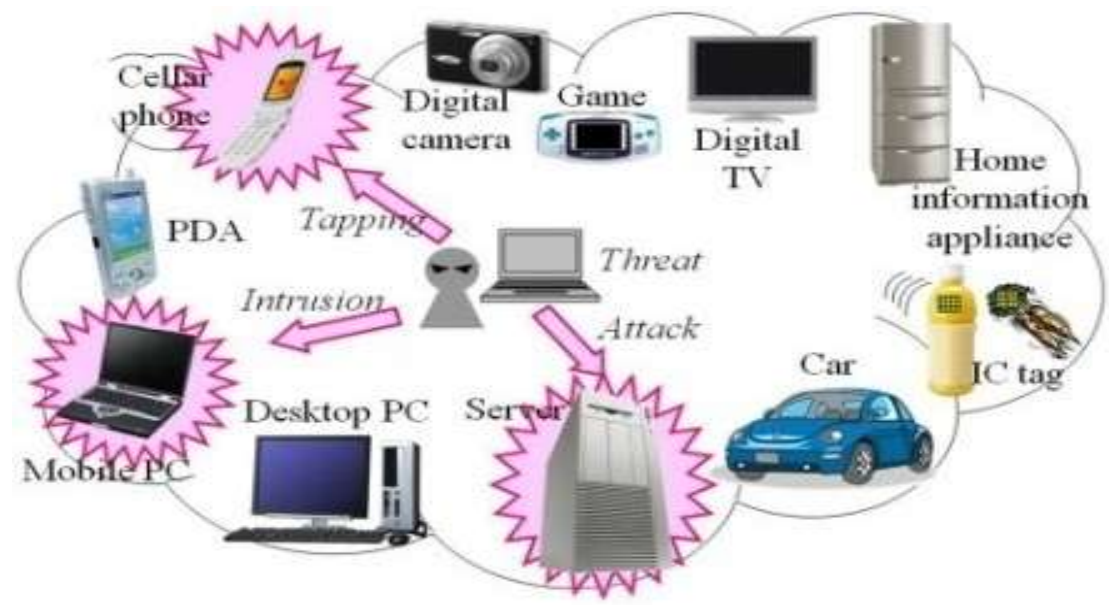




\subsection{Industry sector}

Ad hoc network is widely used for commercial applications. Ad hoc network can also be used in emergency situation such as disaster relief. The rapid development of non-existing infrastructure makes thread hoc network easily to be used in emergency situation.

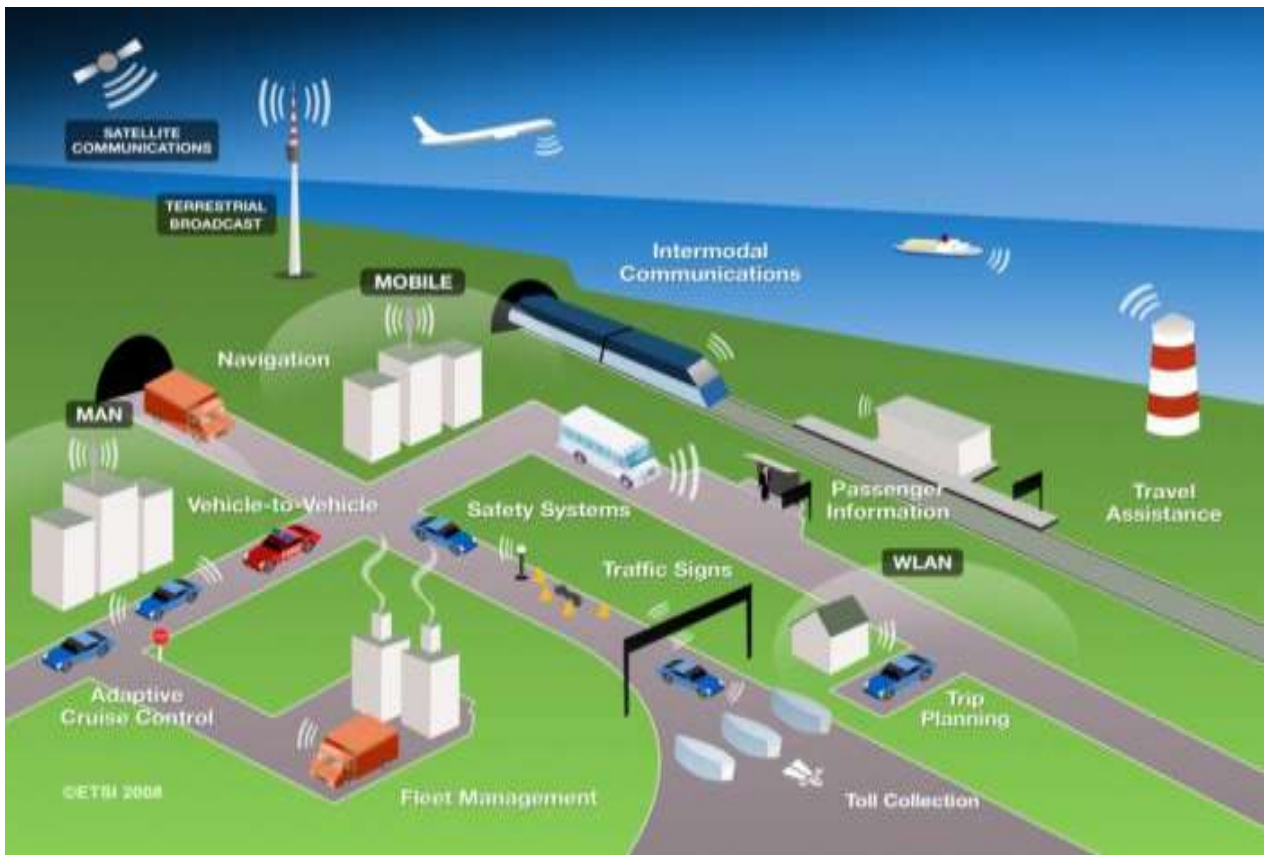

\section{Advantage Of Ad Hoc Mobile Network}

There are many reasons better to use ad hoc than infrastructure. The biggest ad-hoc strength is its independency from any infrastructure. Therefore, it is possible to establish an ad hoc network in any difficult situations. The following are the advantages of ad hoc networks.

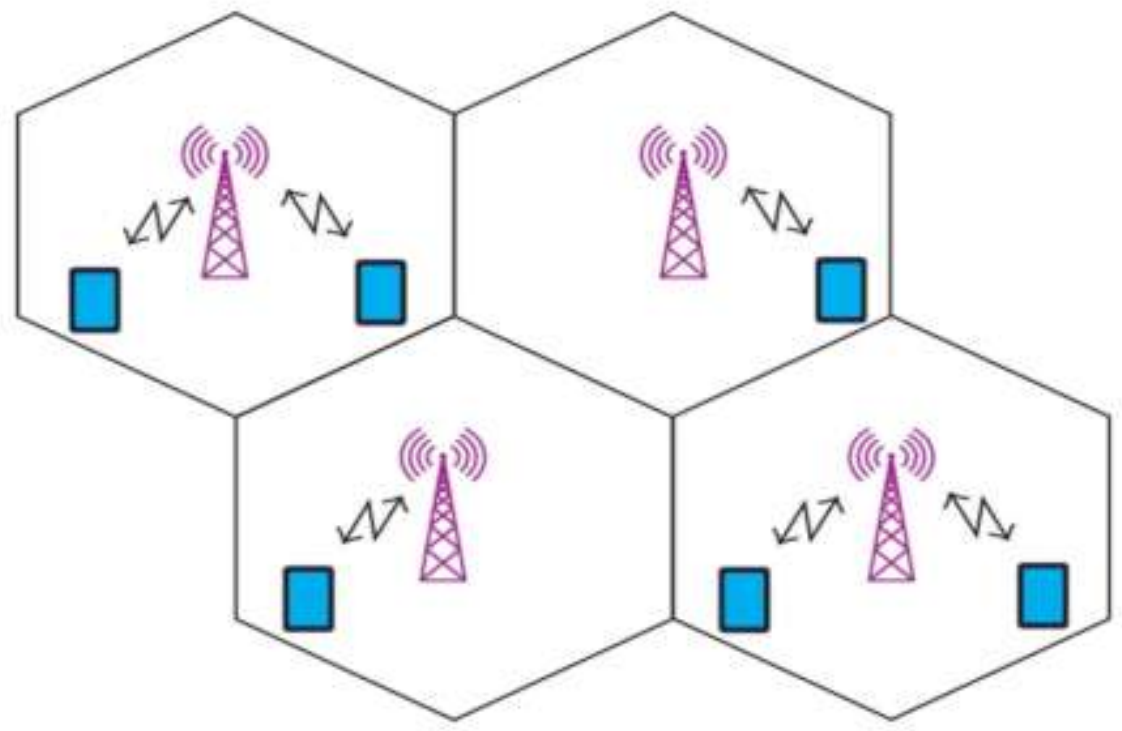

\subsection{No infrastructure and lower cost}

There are situations, with which a user of a communication system cannot rely on an infrastructure. Using a service from a infrastructure can be expensive for specific applications. In an area with very low density, like desert, mountain, or isolated area it is not impossible to establish an Infrastructure. But if we compare how often the people there are using service of infrastructure and how many data per day transmitted with cost of installation, maintenance, and repair, it is maybe too expensive. Almost the same problem with military network. It is obviously very useless to build an infrastructure in a battlefield. Aside from cost of installation, the enemy can destroy the infrastructure in short time. An independent from infrastructure network is needed for both cases. 


\subsection{Mobility (MANET only)}

In the next generation of wireless communication systems, there will be a need for the rapid deploymentof independent mobile users. The most popular examples include military networks, emergency / rescue operations, disaster effort. In these scenarios we can't rely on centralized connectivity. MANETs support nodes' mobility. We can still communicate with our mobile devices as long as the destination is reachable.

\subsection{Decentralized and robust}

Another advantage of ad hoc networks is that they are inherently very robust. Imagine that for some reason one of the base stations is not working. In this case, all users of that base station will lose connectivity to other networks. In the ad hoc networks you can avoid such problem. If one node leaves the network or is not working, you can still have connectivity to other nodes and maybe you can use these nodes to multi-hop your message to the destination nodes, as long as there is at least one way to desired node.

\subsection{Easy to build and spontaneous infrastructure}

Malfunction of a network infrastructure is sometimes not avoidable. It is obviously difficult to repair or replace the malfunction infrastructure in short time, while the network's existence must be maintained all-time. Establishing an ad hoc is a good deal in such situation. The network participants can act as ad hoc nodes and hop the messages.

\section{Challenges In Ad Hoc Networking}

The ad hoc networks are self-forming, self - maintaining, self-healing architecture. The challenges are, no fixed access point, dynamic network topology, contrary environment and irregular connectivity. Ad hoc network immediately forms and accommodate the modification and limited power. Finally, ad hoc have no trusted centralized authority. Due to the dynamic changing property, the ad hoc faces some challenges which are listed in the below sections.

\subsection{Quality of Service (QoS)}

The ad hoc network is dynamically creating the organization whenever the node wants to communicate with their neighbor node. Due the dynamic changing topology in ad hoc network, providing QoS is a tedious task. QoS are essential because of rapid development in mobile technology and real time applications like multimedia, voice.

Providing QoS in ad hoc network is necessary to maintain best-effort-of service. The QoS metric are bandwidth, latency, jitter and delivery guarantee. The bandwidth is used to denote the data rate carried in the network. Latency ensures the delay occur from origin to target. Jitter denotes the variation of delay. Reliability demonstrate the percentage of deny to access the network service. Wireless channels are varying rapidly and it severely affects the multi - hop flows. In ad hoc networks, the peer - to - peer channel quality may alter rapidly. So, the link quality may affect the peer - to - peer QoS metrics in the multi - hop path. The factors of QoSare described below:

\subsubsection{Packets are tangle for the shared media on adjacent links of a flow}

Tangle between the packets in the same stream at various nodes. Such taglines occurs in the wireless channel which is shared by nodes in the neighbor.

\subsubsection{Interference between the nodes affects the transmission quality}

In ad hoc network, QoS is affected by radio interference. Interference strongly arises in ad hoc because nodes are beyond the transmission range. While the nodes are in the transmission, the remaining power is enough to interfere with transmission interference in non - neighboring nodes may drop the packet.

\subsubsection{Limited resources}

Due to the dynamic changing of link flow, ad hoc network provide varying resources. The admission control take decision about whether the available bandwidth is enough for link flow in available resources. Ad hoc networks providing finite bandwidth capacity may affect the end quality of service.

\subsubsection{Highly dynamic}

Ad hoc network characteristics are dynamic changing topology and this dynamic changing occurs due to radio transmission and mobility. 


\subsection{QoS model}

The QoS model specifies the entire architecture of ad hoc network and also maintains the services provided to the network. It does not specify any protocols or implementation. Two types of QoS model are available. One is Integrated Service which must maintain the virtual circuit connection oriented state information in every flow. Second, is Differentiated Service which does not specify any state information.

\subsubsection{QoS signaling}

QoS signaling is used to allocate and reallocate the resources during the network flow. The signaling process is based on two categories. First, QoS signaling is used between the routers. Second, the signaling can be rightly identified and used by the corresponding resources.TheQoS signal can be classified into in-band and out-band. In in-band signaling, signal in formation is carried along with its packet. In out-bound signaling, explicit packet is needed to handle the signaling.

\subsubsection{QoS routing}

QoS routing is designed to provide the optimal path between end-to-end resources that meet QoS requirements such as bandwidth, delay etc. The QoS can maintain ad hoc network by using the admission control to check whether the local available bandwidth is greater than the needed bandwidth. Thus, the process may leads to maintain the quality of service in ad hoc network.

\subsection{Scalability}

The scalability problem occurs in ad hoc networks due to the nature of the multi-hop. The scalability in ad hoc network depends on the network size and forwarded packet capacity in the network. Recently, lot of problems address in large-scaled ad hoc networks. The scalability depends on the following factors:

\subsubsection{Equal node priority}

All the nodes in the network have same priority to share the physical resources. So, all nodes have same data rate.

\subsubsection{Uniform distribution of destination}

The packets are distributed equally from source to $\mathrm{N}-1$ nodes.

\subsubsection{Spreading the node reliably}

The transmission capacity of the node should be large enough than their neighbor nodes to avoid interference.

\subsubsection{Shortest path}

The packets transmitted from source destination depend on the shortest path.The scalability can preserve by dividing the network area according to their geographical location. The whole network area can be divided into small virtual grid cells such that every node in each virtual grid cell can communicate with other nodes in the same cell.

\section{Security}

Security is a major concern in the ad hoc networking standards Data transformation in ad hoc network must be done in a secured way. The security issue in ad hoc network is dynamic topology, bandwidth, small device size and limited battery life. Due to the dynamic nature, it is difficult to maintain secured transmission in the network. The ad hoc network does not depend on any pre-existing infrastructure so that the node can leave and join the network in such a situation where security may fall down. Two types of attack occur in ad hoc network, first is passive attack, this attack does not change the transmitted data in the network. But, it can allow unauthorized user to discover the message. Second, is active attack, it is a severe attack and prevents the message flow between the node in the network. It may allow the unauthorized user to modify the message. The malicious node can be identified by dropped packet, battery drained, bandwidth consumption, unreliable packets, delay, connection break and false routing.

\subsection{Security approach Scope}

It describes whether the node is available to provide the services. It also provides facility to access the authorized user in a desired time.

\subsubsection{Confidentiality}

It ensures only authorized user can access the information. It should be protected against unauthorized user and snooping. 


\subsubsection{Incorruptibility}

It must provide the assurance that the transformation of message without any corruption. Authorized user only has the privilege to access and modify the data. It also certifies when and where the message was delivered.

\subsection{Verification}

It describes participant nodes which are authenticated in the communication network. The resources in the network must communicate through authenticated nodes.

\subsection{Approval}

The authorization assigns different access rights to different types of users. For example, a network management can be performed by network administrator only.

\subsection{Aggression}

It can preserve the network functionality when the parts of nodes are fragmented.

\subsection{Uniqueness}

It ensures that malicious node does not resend previously captured packets.

\section{Power Control}

Power control is one of the main components in ad hoc network as battery provides limited capacity to the nodes. If the power failure occurs in the node, it may affect itself and the entire network architecture gets fragmented. The reason for power control is limited capacity of nodes, non-static infrastructure, constraints on the battery sources, selection of excellent transmission power and channel utilization. Ad hoc power maintenance is based on:

\subsection{Low capacity condition}

The low power mode enables the node to be active during transmission of packet and it makes the node inactive when they are waiting to receive the packet.

\subsection{Transmission power control}

The transmission power control must be based on transmission range, error rate and interference. Thus, the strong transmission power increases the transmission range and reduces the hop count to the destination.

\subsection{Power aware routing}

The routing protocols are designed to find the shortest hop between source and destination. Thus, the routing protocol allows increasing the network life time by reducing the usage of nodes battery life time. In order to maintain power control by minimizing the active energy, the power down mode is used to minimize energy during inactive. The load balancing technique allows the network maximizing by reducing over usage of the node.

\section{Cooperation Between Nodes}

Cooperation between the nodes is essential in the ad hoc network. Each node in the network cooperates with other node for packet forwarding and routing. Due to dynamic changing topology, the nodes in the network do not rely on other node. In ad hoc network, source node transmits the data to destination via intermediate node. Without cooperation between the neighboring nodes source, node cannot transfer the data successfully. Therefore, frequent modification in link may lead for uncertain topology changes and network disconnections.

The non-cooperative nodes may be described in two ways, first one is the malicious nodes refer that the set of the node attack the network or break the link. The selfish nodes use the network for their purpose without pay back for the usage of network. Both nodes are considered as misbehaving nodes. In ad hoc high level cooperation between the neighbor nodes make the network more flexible. Low level cooperation among the node makes the network vulnerable. Strategies for node cooperation are:

\subsection{Concentrating acquaintance}

The node, forward or drop the packet based on the neighbor node behavior. This requires the node to listen in abandoned mode to capture the actions of its neighbor node.

\subsection{Conversation of node}

Nodes are always communicated with one of nearest neighbor node in the entire network. It must identify the misbehavior node and isolate the corresponding node. To manage the cooperation between nodes, it is needed to optimize the trade-off of forwarding packets for others vis-à-vis conserving their energy resources. 


\section{Conclusion}

The rapid evolution in the field of mobile computing is driving a new alternative way for mobile communication, in which mobile devices form a self-creating, self -organizing and self -administering wireless network, called a mobile ad hoc network. Its intrinsic flexibility, lack of infrastructure, ease of deployment, auto-configuration, low cost and potential applications makes it inessential part of future pervasive computing environments. As a consequence, the seamless integration of mobile ad hocnetworks with other wireless networks and fixed infrastructures will be an essential part of the evolution towards future fourth-generation communication networks. Froma technological point of view, the realization of this vision still requires a large number of challenges to be solved related to devices, protocols, applications and services. The concise discussion in this paper shows that, despite the large efforts of the MANETresearch community and the rapid progress made during the last years, a lot of challenging technical issues remains unanswered. Appropriate business scenarios, applications and economical models need to be identified, together with technological advances, making a transition of ad hoc networks to the commercial world viable.

\section{References}

[1]. C. C. Chiang, T. C. Tsai, W. Liu and M. Gerla, Routing in clustered multihop, mobile wireless networks with fading channel, The Next Millennium, The IEEE SICON, 1997.

[2]. B. Bellur, et. al, "Topology Dissemination Based on Reverse-Path Forwarding (TBRPF)", IETF Internet Draft, draft-ietf-manettbrpf-08.txt, April 2003.

[3]. Stefano Basagni, IrnrichChlamtac, Violet R. Syrotiuk and Barry A. Woodward, “ A Distance Routing Effect Algorithm for Mobility (DREAM)," in Proceedings of the 4th annual ACM/IEEE international conference on Mobile computing and networking texas, United States, pages :76-84 in 1998.

[4]. M. Abolhasan, B. Hagelstein, J. C.P. Wang, "Real-world performance of current proactive multi-hop mesh protocols", IEEE APCC, Shanghai, China, 8-10th October 2009.

[5]. Haas ZJ, Pearlman MR, Samar P, "The Zone Routing Protocol (ZRP) for Ad Hoc Networks", IETF draft, July 2002, available at http://tools.ietf.org/id/draft-ietf-manetzone-zrp-04.txt. Accessed 21 February 200894 A.-S.K. Pathan and C.S. Hong

[6]. D. D. Perkins, H. D. Hughes, and C. B. Owen, "Factors affecting the performance of ad hoc networks," in Proc. of IEEE International Conference on Communications (ICC) 2002, vol. 4, pp. 2048-2052, 2002. J.

[7]. MehranAbolhasan , TadeuszWysocki, and ErykDutkiewic, "A review of routing protocols for mobile ad hoc networks," Ad Hoc Networks 2 (2004) 1-22, www.elsevier.com/locate/adhoc

[8]. A. Boukerche, "Performance comparison and analysis of ad hoc routing algorithms," in Proc. of IEEE International Conference on Performance, Computing, and Communications, pp. 171-178, 2001.

[9]. B. Bellur, R. G. Ogier, and F. L. Temlin," Topology Dissemination Based on Reverse-Path Forwarding (TBRPF)", RFC 3684, February 2004.

[10]. N. Nikanein, C. Bonnet, and N. Nikaein, "Hybrid Ad Hoc Routing Protocol - HARP," in proceeding of IST 2001: International Symposium on Telecommunications http://www.eurecom.fr/ nikaeinn/harp.ps

[11]. Hai-KeongToh, "Associativity-Based Routing for Ad-Hoc Mobile Networks in Wireless Personal Communications," An International Journal Volume 4 , Issue 2 (March 1997) Pages: 103 - 139.

[12]. Tai Hieng Tie, Chong Eng Tan and Sei Ping Lau, "Alternate Link Maximum Energy Level Ad-hoc Distance Vector Scheme for Energy Efficient Ad-hoc Networks Routing," in International Conference on Computer and Communication Engineering (ICCCE 2010), 11-13 may 2010, Kuala Lumpur, Malaysia.

[13]. Young-BaeKo and Nitin H. Vaidya, "Location-Aided Routing (LAR) in mobile ad-hoc networks in Wireless Networks," Volume 6, Pages: 307 - 321 Issue 4 (July 2000).

[14]. M.S. CORSON AND A. EPHREMIDES, "Lightweight Mobile Routing protocol (LMR), A distributed routing algorithm for mobile wireless networks, Wireless Networks 1 (1995).

[15]. M. Geria, Guangyu, X. Hong, and T. Chen, "Fisheye State Routing Protocol (FSR) for Ad Hoc Networks Internet Draft," http://tools.ietf.org/html/draft-ietf-manet-fsr, work in progress, June 2001.

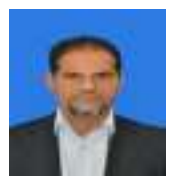

Ahlal H Montaser Mohamed PhD in the Department of Computing at University of Bradford,UK( 2004 - 2008 ).BSc at Department of control engineering, Faculty of Electronic Engineering, Baniwalid, Libya(1991-1999 ). MSc Computing at at University of Bradford, UK. (2002-2003). Staff Member inFaculaty IT University of Tripoli (2016).

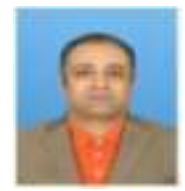

Amamer Khalil Masoud Ahmidat $\mathrm{PhD}$ in the computer and information Faculaty of electrical Engand information technical University of Kosice Slovaki (2003-2008) . BSc electronic .Eng Computer Dept (1987-1991).MSc in computer engineering and information Faculaty of electrical Eng.and information technical University of Kosice Slovaki (1995-1998). University Staff Member in Faculaty of Science in Baniwalid, Assistant Professor from ( 01-10-2013.)

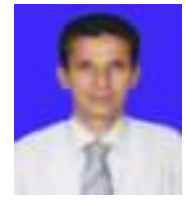

Alhamali Masoud Alfrgani .Ali ph.D in computer engineering and information From Sam Higginbotom university of Agriculture, Technology \& Sciences (2017).B.tech degree in computer engineering from Technology College of Civil Aviation \& Meterology In year 1992 Tripoli -Libya .Mtech. From Sam Higginbotom university of Agriculture, Technology \& Sciences in year 2012 